\title{
Hydrogen Sulfide Inhibits Fruit Softening by Regulating Ethylene Synthesis and Signaling Pathway in Tomato (Solanum lycopersicum)
}

Kang-Di Hu, Xiao-Yue Zhang, and Sha-Sha Wang School of Food and Biological Engineering, Hefei University of Technology, Hefei 230009, China

Jun Tang, Feng Yang, and Zhong-Qin Huang Xuzhou Institute of Agricultural Sciences of the Xuhuai District of Jiangsu Province, Xuzhou 221131, China

Jing-Yu Deng, Si-Yuan Liu, and Shang-Jun Zhao School of Food and Biological Engineering, Hefei University of Technology, Hefei 230009, China

Lan-Ying Hu

School of Food and Biological Engineering, Hefei University of Technology, Hefei 230009, China; Anhui Province Key Laboratory of Functional Compound Seasoning, Anhui Qiangwang Seasoning Food Co., Ltd., Jieshou 236500, China

Gai-Fang Yao and Hua Zhang School of Food and Biological Engineering, Hefei University of Technology, Hefei 230009, China

Additional index words. cell wall-degrading enzymes, postharvest storage, gene expression, correlation analysis, fruit firmness

Abstract. Hydrogen sulfide $\left(\mathrm{H}_{2} \mathrm{~S}\right)$ has been proven to be a multifunctional signaling molecule in plants. In this study, we attempted to explore the effects of $\mathrm{H}_{2} \mathrm{~S}$ on the climacteric fruit tomato during postharvest storage. $\mathrm{H}_{2} \mathrm{~S}$ fumigation for $1 \mathrm{~d}$ was found to delay the peel color transition from green to red and decreased fruit firmness induced by ethylene. Further investigation showed that $\mathrm{H}_{2} \mathrm{~S}$ fumigation downregulated the activities and gene expressions of cell wall-degrading enzymes pectin lyase (PL), polygalacturonase (PG), and cellulase. Furthermore, $\mathrm{H}_{2} \mathrm{~S}$ fumigation downregulated the expression of ethylene biosynthesis genes $S I A C S 2$ and $S I A C S 3$. Ethylene treatment for $1 \mathrm{~d}$ was found to induce the expression of $\mathrm{SlACO1}, \mathrm{SlACO3}$, and $\mathrm{SlACO4}$ genes, whereas the increase was significantly inhibited by $\mathrm{H}_{2} \mathrm{~S}$ combined with ethylene. Furthermore, $\mathrm{H}_{2} \mathrm{~S}$ decreased the transcript accumulation of ethylene receptor genes SIETR5 and SIETR6 and ethylene transcription factors $S I C R F 2$ and $S I E R F 2$. The correlation analysis suggested that the fruit firmness was negatively correlated with ethylene biosynthesis and signaling pathway. The current study showed that exogenous $\mathrm{H}_{2} \mathrm{~S}$ could inhibit the synthesis of endogenous ethylene and regulate ethylene signal transduction, thereby delaying fruit softening and the ripening process of tomato fruit during postharvest storage.

Tomato (Solanum lycopersicum L.) is an important fruit for human consumption because it provides nutrients like flavor compounds, vitamins, and fiber (Beecher, 1998). Postharvest ripening and senescence involve changes in metabolism and gene expression, which further affect sensory attributes, flesh texture, fruit nutritional quality, and market acceptance. Fruit ripening and senescence are accompanied by texture softening, which is one of the most important factors regarding fruit quality and consumer acceptability (Giovannoni, 2007; Grierson et al., 1986; Musse et al., 2009). Fruit softening is a complex process that results from loosening of the cell wall. Many enzymes such as pectinmethylesterase, polygalacturonase, cellulase, and pectin lyase catalyze the modification and degradation of cell wall components, including pectin, hemicellulose, and others (Fischer and Bennett, 1991; Gwanpua et al., 2016; Thompson et al., 1998). Therefore, delaying fruit softening will maintain the texture of fruit and improve the market values.

Ethylene is an important phytohormone in plants and is involved in many aspects of the plant life, including seed germination, root hair development, flower senescence, and fruit ripening (Sisler and Yang, 1984). To- mato, as a typical respiratory climacteric fruit, ripening and senescence are largely dependent on endogenous production and ethylene action (Liu et al., 2015). Ethylene is generated from the precursor of methionine through Yang's cycle. Studies of ethylene biosynthesis have been focused on the isolation and characterization of ACS (1aminocyclopropane-1-carboxylate synthase) and ACO (1-aminocyclopropane-1-carboxylate oxidase) genes, and ACC (1-aminocyclopropane-1carboxylate) is the immediate precursor of ethylene (Adams and Yang, 1979; Alexander and Grierson, 2002; Wang et al., 2002). After synthesis, ethylene is perceived by ethylene receptors (ETRs) (Payton et al., 1996). Subsequently, a signaling cascade including both positive and negative regulators modulates a large family of transcription factors called ethylene response factors (ERF) (Thirugnanasambantham et al., 2015). Enormous studies demonstrated that the transcription factor ERF in the AP2/ERF family modulates metabolic pathways involved in fruit ripening and senescence, chlorophyll degradation, fruit softening, and changes in aromas (De Boer et al., 2011; Licausi et al., 2013; Qi et al., 2011). Because ethylene is the key phytohormone regulating fruit ripening, many studies have focused on delaying the action or synthesis of ethylene. For instance, ethylene antagonist 1-methylcyclopropene (1-MCP) was broadly applied to delay fruit ripening and senescence (Song et al., 2018; Watkins et al., 2000). Nitric oxide (NO), an important signaling molecule, was found to delay fruit softening in mango fruit during storage by inhibiting ethylene biosynthesis (Zaharah and Singh, 2011).

Hydrogen sulfide $\left(\mathrm{H}_{2} \mathrm{~S}\right)$ is found to be the third most important gasotransmitter in animals and plants after NO and carbon monoxide (CO) (Gadalla and Snyder, 2010; Lisjak et al., 2010; Wang, 2010). $\mathrm{H}_{2} \mathrm{~S}$ participates in almost all aspects of plant life, including seed germination, root organogenesis, stomatal movement, resistance to stress conditions, and plant senescence (Chen et al., 2011; Qiao et al., 2015; Wang et al., 2012; Zhang et al., 2009). Similar to the role of NO in the regulation of fruit ripening and senescence, accumulating studies found that $\mathrm{H}_{2} \mathrm{~S}$ could alleviate postharvest ripening and senescence by modulation of the antioxidant system of kiwifruit, apple, banana, and others (Gao et al., 2013; Ge et al., 2017; Hu et al., 2012). Whether and how $\mathrm{H}_{2} \mathrm{~S}$ is involved in the ripening and senescence of tomato are still unclear. Therefore, in this study, we attempted to illuminate the mechanism of $\mathrm{H}_{2} \mathrm{~S}$ in modulating tomato ripening and senescence. Additionally, the impact of $\mathrm{H}_{2} \mathrm{~S}$ on the ethylene synthesis and signaling pathway in tomato fruit ripening was investigated.

\section{Materials and Methods}

Plant materials and sample preparation. Tomatoes (Solanum lycopersicum cv. Micro Tom) were harvested at the mature green stage; plants were grown in the greenhouse at Hefei University of Technology in Hefei, China. The tomato plants were cultivated at 
$24{ }^{\circ} \mathrm{C}$ with 16 -h day and 8-h night conditions, with relative humidity at $50 \%$ to $65 \%$. The media for culturing tomato seedlings was composed of nutritional soil, peat soil, and vermiculite in a ratio of $3: 5: 1$. Thirty days post anthesis tomato fruits, which is in the mature-green stage, with similar size and without physical injuries or infections were chosen and stored in containers. The tomatoes were fumigated with ethylene $\left(\mathrm{C}_{2} \mathrm{H}_{4}\right)$ released from $100 \mathrm{~mL}$ of $1 \mathrm{~g} \cdot \mathrm{L}^{-1}$ ethephon solution or co-treatment of $\mathrm{C}_{2} \mathrm{H}_{4}+\mathrm{H}_{2} \mathrm{~S}$ released from $150 \mathrm{~mL}$ of $1 \mathrm{mmol} \cdot \mathrm{L}^{-1}$ sodium hydrosulfide (NaHS) solution and $100 \mathrm{~mL}$ of $1 \mathrm{~g} \cdot \mathrm{L}^{-1}$ solution ethephon at $23 \pm 0.5^{\circ} \mathrm{C}$ with relative humidity of $85 \%$ to $90 \%$. After $1 \mathrm{~d}$ of storage, the solutions were replaced with distilled water. Each treatment unit comprised six tomatoes, and each experiment was repeated three times. The flesh of fruit without seeds was randomly sampled every other day and stored at $-80^{\circ} \mathrm{C}$ for subsequent experiments. The changes in tomato fruit color were measured with a color difference meter (model WSC-100; Konica Minolta, Tokyo, Japan), which provided CIE L*, a*, and $\mathrm{b}^{*}$ values, where $\mathrm{L}^{*}$ indicates lightness, $\mathrm{a}^{*}$ indicates chromaticity on a green $(-)$ to red $(+)$ axis, and $b^{*}$ indicates chromaticity on a blue $(-)$ to yellow $(+)$ axis. Each fruit was measured at four equidistant points around the middle area.

Measurement of fruit firmness. The tomato fruit firmness was measured using a 2-mm-diameter flat probe with a texture analyzer (Model TA XT plus; Stable Micro Systems, Surrey, UK). Fruit firmness value was an average of 10 replicates with standard deviation.

Activity assay of cellulase, polygalacturonase, and pectin lyase. Frozen tissues (1 g) of tomato were homogenized with $4 \mathrm{~mL}$ of $8.8 \% \mathrm{NaCl}$ containing $10 \mathrm{~g} \cdot \mathrm{L}^{-1}$ polyvinyl pyrrolidone (PVPP). The mixture was centrifuged at $10,000 g_{\mathrm{n}}$ for $20 \mathrm{~min}$, and the supernatant was collected for enzyme activity assay.

Cellulase activity was determined as described by Abeles and Biles (1991). One unit

Received for publication 10 June 2019. Accepted for publication 16 July 2019 .

Funding for this work was provided by the National Natural Science Foundation of China (31670278, 31970200, 31970312, 31901993, 31872078), the Anhui Provincial Science and Technology Major Project (16030701073), the Earmarked Fund for the China Agriculture Research System (CARS-10-B1), the Fundamental Research Funds for the Central Universities (JZ2018HGTB0241, JZ2018HGBZ0160), the Natural Science Foundations of Anhui Province (1908085MC72), the Key Research and Development Program of Anhui Province (201904a06020031), and National Undergraduate Training Programs for Innovation of China (No. 201810359054, 2018CXCYS195).

K.-D.H., X.-Y.Z., and S.-S.W. contributed equally to this work.

G.-F.Y. and H.Z. are the corresponding authors. E-mail: yaogaifang@hfut.edu.cn or hzhanglab@hfut.edu.cn.
(U) of activity was defined as $1 \mu \mathrm{g}$ of reducing sugar generated per $1 \mathrm{~g}$ of fresh weight (FW) per hour. The cellulase activity was expressed as U.g ${ }^{-1} \mathrm{FW}$.

Polygalacturonase activity was determined according to Pathak and Sanwal (1998). One U of activity was defined as $1 \mu \mathrm{g}$ of galacturonic acid generated per $1 \mathrm{~g}$ of $\mathrm{FW}$ per hour. The activity of polygalacturonase was expressed as $\mathrm{U} \cdot \mathrm{g}^{-1} \mathrm{FW}$.

Pectin lyase was determined following the method described by Pitt (1988). The reaction was performed in a mixture of $1 \mathrm{~mL}$ of $1 \%$ pectin solution and $0.5 \mathrm{~mL}$ of enzyme extraction at $40{ }^{\circ} \mathrm{C}$ for $5 \mathrm{~min}$ and ended by adding $3.5 \mathrm{~mL}$ of $0.01 \mathrm{~mol} \cdot \mathrm{L}^{-1} \mathrm{HCl}$. The absorbance was measured at $235 \mathrm{~nm}$, and $1 \mathrm{U}$ of activity was defined as an absorbance increase of 1.0 per min. The activity of pectin lyase was expressed as U.g ${ }^{-1} \mathrm{FW}$.

Quantitative reverse-transcription polymerase chain reaction analysis. Candidate genes encoding cell wall-degrading enzymes, ethylene synthesis, and signaling genes were identified using a hidden Markov model search of protein domains obtained from the Pfam database (http://pfam.xfam.org/) and a local BLASTN analysis of genes (https:// solgenomics.net/).

Total RNA from $0.1 \mathrm{~g}$ of frozen tomato fruit samples was extracted using the
RNA Extraction Kit (Tiangen, Beijing, China). Then, cDNA was synthesized using a reverse-transcription kit (PrimeScript RT Master Mix; Takara, Kyoto, Japan). The cDNA products were used for the quantitative polymerase chain reaction (qPCR) performed using Bio-Rad IQ5 (Hercules, CA) in a $10-\mu \mathrm{L}$ reaction containing $5 \mu \mathrm{L}$ of $2 \times$ SYBR Premix Ex Taq (Takara, Kyoto, Japan), $0.8 \mu \mathrm{L}$ of cDNA, $0.4 \mu \mathrm{L}$ each for the forward and reverse primers, and $3.4 \mu \mathrm{L}$ of $\mathrm{ddH}_{2} \mathrm{O}$. The specific primers used for the qPCR were designed based on the coding sequence of the genes as shown in the SGN database (https://solgenomics.net/) (Table 1). The qPCR was initiated using a denaturation step at $95{ }^{\circ} \mathrm{C}$ for $60 \mathrm{~s}$, followed by 40 cycles of $95^{\circ} \mathrm{C}$ for $10 \mathrm{~s}, 60^{\circ} \mathrm{C}$ for $30 \mathrm{~s}$, and $72^{\circ} \mathrm{C}$ for $10 \mathrm{~s}$. Expression of the actin gene in control tomatoes was used for normalization of data. The relative expression levels of all genes were calculated and analyzed by the $2^{-\Delta \Delta \mathrm{Ct}}$ method. All analyses were repeated three times using three technical replicates with error bars.

Statistical analysis. Data were based on three replicates in each experiment, and the experiments were repeated independently three times. Statistical significance was tested using a one-way analysis of variance with IBM SPSS Statistics (SPSS version 20.0;

Table 1. Primers used for the quantitative polymerase chain reaction.

\begin{tabular}{|c|c|c|}
\hline Primer & Sequence & Gene ID \\
\hline SILAT56 (forward) & 5'-GCACTCGGGACAGCAATAAA-3' & Solyc03g058910 \\
\hline SILAT56 (reverse) & 5'-GTCACATCTCCAGCACTTGTC-3' & \\
\hline SlLAT59 (forward) & 5'-ATTAGGTTGCACCAGGAGATG-3' & Solyc03g058890 \\
\hline SILAT59 (reverse) & 5'-GAGGGTTATACCAGCACCTTTAG-3' & \\
\hline SIPMEU1 (forward) & 5'-CTCAAGGAAGAACTGACCCTAAC-3' & Solyc03g123630 \\
\hline SIPMEU1 (reverse) & 5'-TTCTGCACCGGTCTCAAATC-3' & \\
\hline SIPG2 (forward) & 5'-GTGGTCCAGGTCATGGTATAAG-3' & Solyc $10 \mathrm{~g} 080210$ \\
\hline SIPG2 (reverse) & 5'-CCTGCCAAGTCTTGATCCTAA-3' & \\
\hline SlACS2 (forward) & 5'-CCACTGGAGCTAATGAGACAAT-3' & Solyc01g095080 \\
\hline SlACS2 (reverse) & 5'-AATGCTGGGTAGTATGGTGAAG-3' & \\
\hline SlACS3 (forward) & 5'-GACTTAGCCATGCGACGTATAA-3' & Solyc02g091990 \\
\hline SlACS3 (reverse) & 5'-CCCACTTGGAGAATGACTTCTT-3' & \\
\hline SlACS6 (forward) & 5'-TGGAGAAAGTGAGAGGAGATAGA-3' & Solyc08g008130 \\
\hline SlACS6 (reverse) & 5'-ACCAGGATCAGCCAAACAA-3' & \\
\hline SlACO1 (forward) & 5'-AAGGAACTAGTGGCAAGTAAGG-3' & Solyc07g049530 \\
\hline SlACOI(reverse) & 5'-TAGGAAGATGGCGCAAGAAG-3' & \\
\hline SlACO3 (forward) & 5'-GATGGAAAGCCCTAGAGTTGAG-3' & Solyc09g089580 \\
\hline SlACO3 (reverse) & 5'-CCTTTAACACCGGCCTTAGT-3' & \\
\hline SlACO4 (forward) & 5'-GCGCCATCTTCCTACTTCTAAT-3' & Solyc02g081190 \\
\hline SlACO4 (reverse) & 5'-CTCAGCCAACTTCTCCAATCT-3' & \\
\hline SlETRl (forward) & 5'-TGTGGAGCAACCCATCTTATC-3' & Solyc $12 \mathrm{~g} 011330$ \\
\hline SIETRI (reverse) & 5'-TCAAGGCCTTTGCAGTAGTC-3' & \\
\hline SIETR2 (forward) & 5'-CCGGAAGTTGCTATTGGAGAT-3' & Solyc $07 \mathrm{~g} 056580$ \\
\hline SIETR2 (reverse) & 5'-ACTGACACACTGCCTTCTTC-3' & \\
\hline SIETR3 (forward) & 5'-CCTTTGGCAAGGATGAGGAATA-3' & Solyc09g075440 \\
\hline SIETR3 (reverse) & 5'-GACAGTTCAGCCCAGTCATTAG-3' & \\
\hline SIETR4 (forward) & 5'-GCCACGGCTATAACCCTTATTA-3' & Solyc06g053710 \\
\hline SIETR4 (reverse) & 5'-CTTCTCTACCAAGATCCCAAGTC-3' & \\
\hline SIETR5 (forward) & 5'-ATGAGAGAGTCTGGAGCTATGA-3' & Solyc11g006180 \\
\hline SIETR5 (reverse) & 5'-AGAACAGTGGCATGGGATAAA-3' & \\
\hline SIETR6 (forward) & 5'-GTGGTGTGGAGGTTCTGTTAG-3' & Solyc09g089610 \\
\hline SIETR6 (reverse) & 5'-AGCACCGAGGCATTCATATC-3' & \\
\hline SIERF1 (forward) & 5'-CGCGTAATGGAATTAGGGTTTG-3' & Solyc $05 \mathrm{~g} 051200$ \\
\hline SlERF1 (reverse) & 5'-CCTCATTGATAATGCGGCTTG-3' & \\
\hline SIERFD2 (forward) & 5'-GGAGATGGTGACGGAGTTTAC-3' & Solyc12g056590 \\
\hline SlERFD2 (reverse) & 5'-CCGAGCTGGACGAAGAATAAG-3' & \\
\hline SIERF2 (forward) & 5'-GTGGTGAGCAGATGAAGAAGAG-3' & Solyc09g075420 \\
\hline SIERF2 (reverse) & 5'-CGAATTTCAGCAGCCCATTTAC-3' & \\
\hline SlTubulin (forward) & 5'-TAGAGCCTGGTACGATGGATAG-3' & Solyc08g006890 \\
\hline SlTubulin (reverse) & 5'-CAACTCAGCGCCTTCAGTATAA-3' & \\
\hline
\end{tabular}


Armonk, NY), and the results were expressed as the means \pm SD. Significant differences were calculated following a significance $(P<$ 0.01 or $P<0.05) t$ test. The Pearson correlation coefficient $(\mathrm{R})$ was used to show the correlation among fruit firmness, cellulase activities, polygalacturonase (PG), pectin lyase (PL), expressions of softening-related genes, ethylene biosynthesis genes, ethylene receptor genes, and ethylene response factors of tomato fruit treated with $\mathrm{C}_{2} \mathrm{H}_{4}$ and $\mathrm{C}_{2} \mathrm{H}_{4}+\mathrm{H}_{2} \mathrm{~S}$. The heatmap of the parameters was drawn by $\mathrm{R}$ script.

\section{Results}

Effects of $\mathrm{H}_{2} \mathrm{~S}$ and ethylene on the ripening and senescence of tomatoes during storage. To evaluate the potential impact of exogenous $\mathrm{H}_{2} \mathrm{~S}$ on fruit ripening, tomatoes at the mature green stage were treated with ethylene or ethylene $+\mathrm{H}_{2} \mathrm{~S}$ for $1 \mathrm{~d}$ and subjected to normal conditions. As shown in Fig. 1A, fruits treated with ethylene showed obvious color breaking at $1 \mathrm{~d}$ and were fully red at $5 \mathrm{~d}$. However, the ripening process was delayed by $\mathrm{C}_{2} \mathrm{H}_{4}+\mathrm{H}_{2} \mathrm{~S}$ co-treatment, and tomatoes at the color breaking stage were still observed on day 5. Changes in the skin color of tomato fruits during storage were evaluated via $L^{*}, a^{*}$, and $b^{*}$. Figure 1B shows a loss of lightness in both treatment fruits, whereas the $\mathrm{L}^{*}$ value of fruits treated with $\mathrm{C}_{2} \mathrm{H}_{4}+\mathrm{H}_{2} \mathrm{~S}$ decreased more slowly and was maintained at a higher value than that of fruits treated with $\mathrm{C}_{2} \mathrm{H}_{4}$. Furthermore, the a* values of fruits treated with $\mathrm{C}_{2} \mathrm{H}_{4}+\mathrm{H}_{2} \mathrm{~S}$ increased slowly and were significantly lower than those of fruits treated with $\mathrm{C}_{2} \mathrm{H}_{4}$ during storage (Fig. 1C), suggesting that the color change from green to red was delayed in tomatoes treated with $\mathrm{C}_{2} \mathrm{H}_{4}+\mathrm{H}_{2} \mathrm{~S}$.

Effects of $\mathrm{H}_{2} \mathrm{~S}$ and ethylene on the fruit firmness of tomatoes during storage. Fruit firmness is an important factor used to evaluate the quality of tomatoes. As shown in Fig. 2A, the firmness of the two groups decreased gradually from day 0 to day 5 during storage. However, ethylene $+\mathrm{H}_{2} \mathrm{~S}$ resulted in significantly higher levels of fruit firmness compared with ethylene treatment during the whole storage period, suggesting that the co-treatment significantly delayed the ethylene-induced softening of tomato fruit.

Effects of $\mathrm{H}_{2} \mathrm{~S}$ and ethylene on the activities of cell wall-degrading enzymes in tomatoes during storage. To explore the role of $\mathrm{H}_{2} \mathrm{~S}$ in delaying tomato fruit softening, the activities of cell wall-degrading enzymes were determined in fruit. Figure $2 \mathrm{~B}$ showed the changes of cellulase activities in tomatoes treated with ethylene and ethylene $+\mathrm{H}_{2} \mathrm{~S}$. Cellulase activity decreased dramatically on day 1 , followed by a significant increase. Furthermore, ethylene $+\mathrm{H}_{2} \mathrm{~S}$ treatment induced significantly low levels of cellulase activity on day 3 and day 5 compared with ethylene treatment. As shown in Fig. 2C, the activities of $\mathrm{PG}$ decreased gradually with both treatments. However, in comparison with the ethylene treatment, the ethylene $+\mathrm{H}_{2} \mathrm{~S}$ co-treatment apparently maintained lower PG activities from day 1 to day 5. Figure $2 \mathrm{D}$ shows that the activities of pectin lyase increased significantly on day 1 , followed by a decrease. The activity of the ethylene $+\mathrm{H}_{2} \mathrm{~S}$ co-treatment group of tomatoes on day 1 was $23.1 \%$ lower than that of ethylene treatment group of tomatoes. From day 3 to day 5 , the pectin lyase activity of tomatoes administered co-treatment was maintained at a significantly lower value $(P<$ $0.01)$ than that of tomatoes treated with ethylene.

Effects of $\mathrm{H}_{2} \mathrm{~S}$ and ethylene on the expression of softening-related genes in tomatoes. Fruit softening is attributable to cell wall degradation caused by related enzymes; therefore, the expressions of softening-related genes are modulated during fruit ripening. As shown in Fig. 3A, the
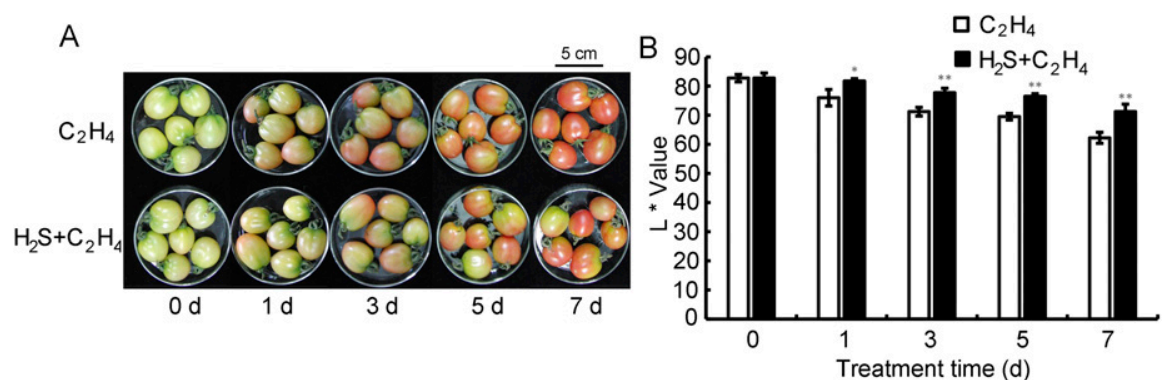

C

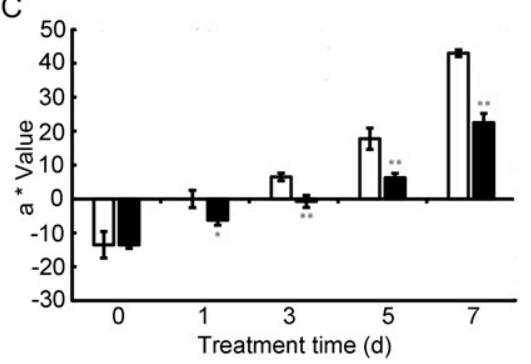

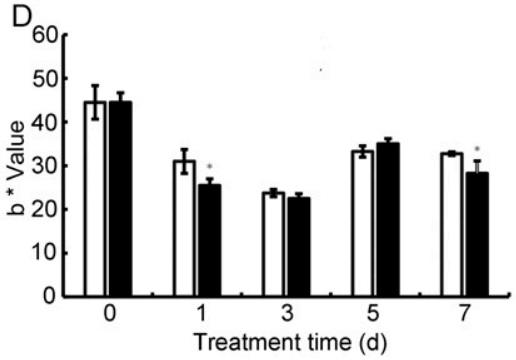

Fig. 1. Color changes in tomato fruit treated with $\mathrm{C}_{2} \mathrm{H}_{4}$ and $\mathrm{C}_{2} \mathrm{H}_{4}+\mathrm{H}_{2} \mathrm{~S}$ for $1 \mathrm{~d}(1 \mathrm{~d}$ of storage) and subsequently stored in normal conditions $\left(3,5\right.$, and $7 \mathrm{~d}$ of storage) $(\mathbf{A})$. $\mathrm{L}^{*}$ indicates lightness $(\mathbf{B}), \mathrm{a}^{*}$ indicates chromaticity on a green $(-)$ to red $(+)$ axis $(\mathbf{C})$, and $b^{*}$ indicates chromaticity on a blue $(-)$ to yellow $(+)$ axis $(\mathbf{D})$.
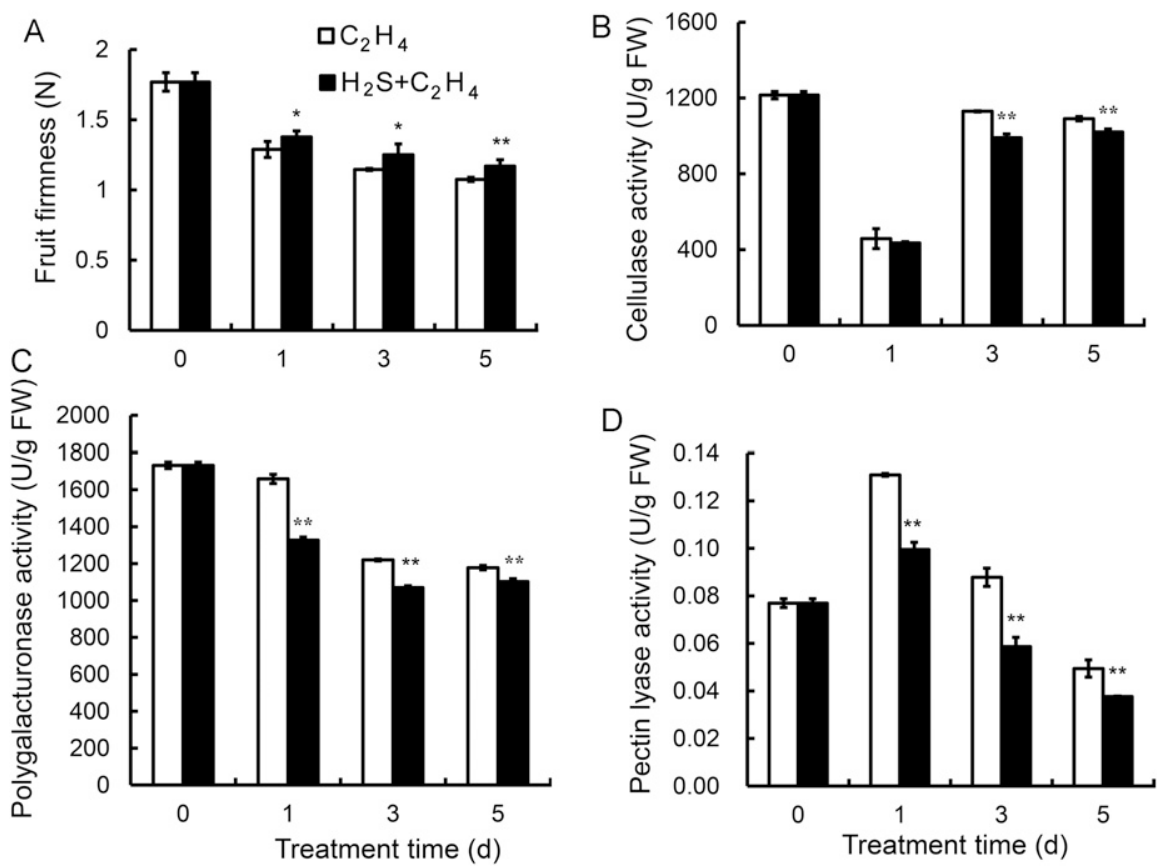

Fig. 2. Effects of $\mathrm{C}_{2} \mathrm{H}_{4}$ and $\mathrm{C}_{2} \mathrm{H}_{4}+\mathrm{H}_{2} \mathrm{~S}$ treatments on the firmness of tomato fruit $(\mathbf{A})$ and the activities of cell wall-degrading enzymes cellulase (B), polygalacturonase (C), and pectin lyase (D). Fruit were fumigated with $\mathrm{C}_{2} \mathrm{H}_{4}$ and $\mathrm{C}_{2} \mathrm{H}_{4}+\mathrm{H}_{2} \mathrm{~S}$ for $1 \mathrm{~d}$ and then stored in normal conditions in a 3- $\mathrm{L}$ chamber. Error bars indicate SE $(n=10$ for fruit firmness and $n=3$ for enzyme activities). Asterisks indicate significant differences between fruit treated with $\mathrm{C}_{2} \mathrm{H}_{4}$ and $\mathrm{C}_{2} \mathrm{H}_{4}+\mathrm{H}_{2} \mathrm{~S}$ according to Student's $t$ test $(* P<0.05 ; * * P<0.01)$. 
transcript level of the pectin lyase gene SILAT56 increased gradually until day 5 with both treatments during the storage of tomatoes, and no significant difference was observed between ethylene and ethylene $+\mathrm{H}_{2} \mathrm{~S}$ treatment. Figure 3B shows the changes in another pectin lyase gene, SILAT59, in tomatoes. The expression of SILAT59 increased slightly on day 1 in tomatoes with both treatments. On day 5 of storage, the transcript of SlLAT59 increased more than two-times that on day 1 with ethylene treatment. Compared with the ethylene group, the expression of SILAT59 on day 5 with ethylene $+\mathrm{H}_{2} \mathrm{~S}$ cotreatment was significantly lower $(P<0.01)$ than that with ethylene treatment.

Figure 3C shows the changes in the expression of the pectin methylesterase gene SIPMEU1 in tomatoes during storage. The expression of SIPMEU1 decreased gradually with both treatments, but no significant difference was observed between them. As shown in Fig. 3D, the expression of the polygalacturonase gene $S I P G 2$ increased dramatically during tomato storage, and it especially increased on day 5. The gene expression of SIPG2 with ethylene treatment was significantly higher than that with the cotreatment on day 1 and day 5; however, an opposite trend was observed on day 3 .

Effects of $\mathrm{H}_{2} \mathrm{~S}$ and ethylene on the expression of ethylene synthesis genes in tomatoes. Ethylene is the predominant phytohormone that promotes fruit ripening; therefore, the expressions of the genes involved in the biosynthesis of ethylene were analyzed. Figure 4 shows the expression patterns of the six ethylene biosynthesis genes, SlACS2, SlACS3, SlACS6, SlACO1, $S l A C O 3$, and SlACO4, in tomatoes treated with ethylene and with ethylene $+\mathrm{H}_{2} \mathrm{~S}$. As shown in Fig. 4A, the expression of SIACS2 increased and peaked on day 3 , followed by a decrease with both treatments. However, ethylene $+\mathrm{H}_{2} \mathrm{~S}$ reduced SlACS2 expression significantly on day 3 compared with ethylene treatment. Figure $4 \mathrm{~B}$ shows that the expression of SlACS3 fluctuated during tomato storage, and a significantly lower level of SlACS3 expression was observed with cotreatment on day 3 in comparison with ethylene treatment alone. Figure $4 \mathrm{C}$ shows that the expression of SlACS6 increased slightly with both treatments and that $\mathrm{H}_{2} \mathrm{~S}$ did not significantly affect the expression. Figure 4D shows that $1 \mathrm{~d}$ of fumigation with ethylene induced an increase of SlACO1, followed by a plateau. However, ethylene $+\mathrm{H}_{2} \mathrm{~S}$ treatment sustained significantly lower levels of SIACO1 expression on day 1, followed by a peak on day 3 . SlACO1 expression with co-treatment was significantly lower on day 1 and day 5 , but it was higher on day 3 compared with ethylene treatment alone. Figure 4E shows that ethylene treatment exhibited a continuous increase in SlACO3 expression, whereas its expression with co-treatment increased slightly and peaked on day 3 , followed by a decrease. Ethylene $+\mathrm{H}_{2} \mathrm{~S}$ induced a significantly lower level of SlACO3 expression on day 1 and day
5 during tomato storage compared with ethylene treatment. Changes in patterns similar to those of SlACO1 were observed for SlACO4 expression during tomato storage. Ethylene induced an increase in SlACO4 expression on day 1 , but the increase was greatly attenuated with ethylene $+\mathrm{H}_{2} \mathrm{~S}$ cotreatment. The expression of SlACO4 with cotreatment was significantly higher on day 3 and lower on day 5 compared with that with ethylene treatment. $\mathrm{H}_{2} \mathrm{~S}$ repressed the expression of ethylene synthesis genes SlACS2, SlACS3, SlACO1, SlACO3, and SlACO4 in tomatoes during storage to different extents.
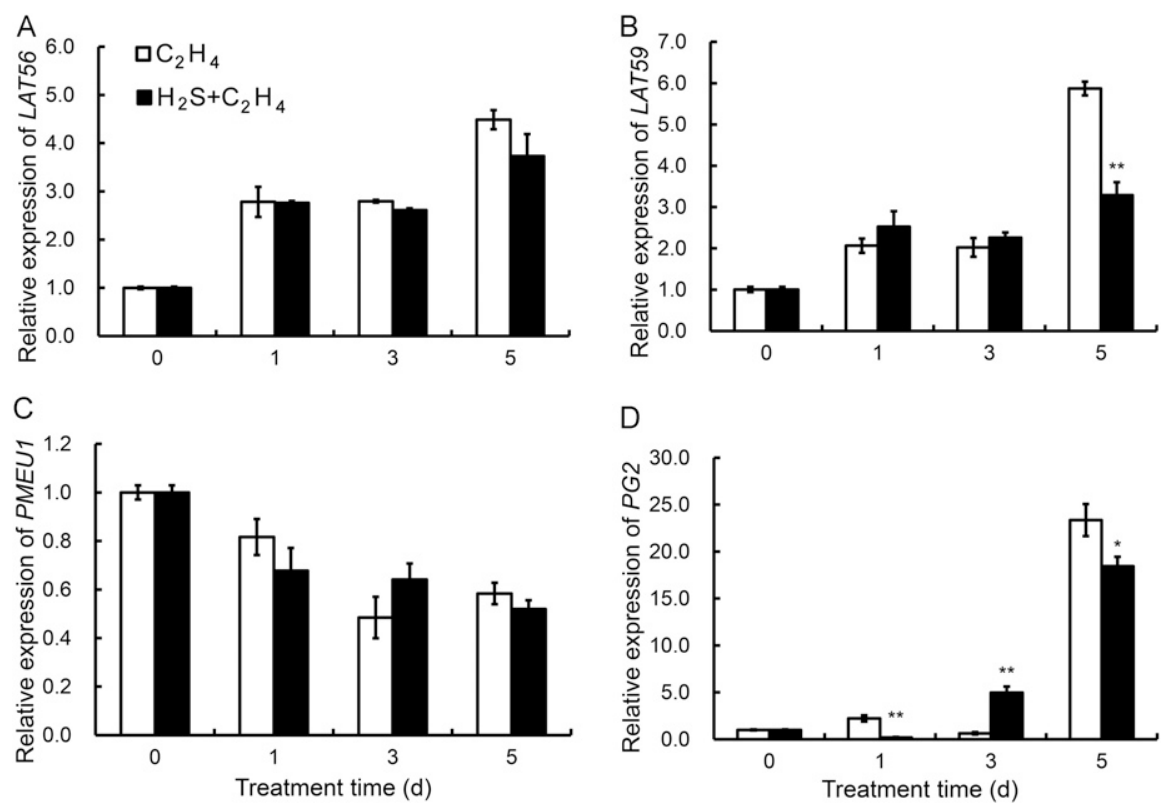

Fig. 3. Relative gene expression patterns of softening-related genes pectin lyases SlLAT56 (A) and SlLAT59 (B) and pectin methylesterase (SIPMEU1) (C) and polygalacturonase (SIPG2) (D) in tomato fruit treated with $\mathrm{C}_{2} \mathrm{H}_{4}$ and $\mathrm{C}_{2} \mathrm{H}_{4}+\mathrm{H}_{2} \mathrm{~S}$ for $1 \mathrm{~d}$. Error bars indicate $\mathrm{SE}(\mathrm{n}=3)$. Asterisks indicate significant differences between fruit treated with $\mathrm{C}_{2} \mathrm{H}_{4}$ and $\mathrm{C}_{2} \mathrm{H}_{4}+\mathrm{H}_{2} \mathrm{~S}$ according to Student's $t$ test (*P<0.05; ** $P<0.01)$.
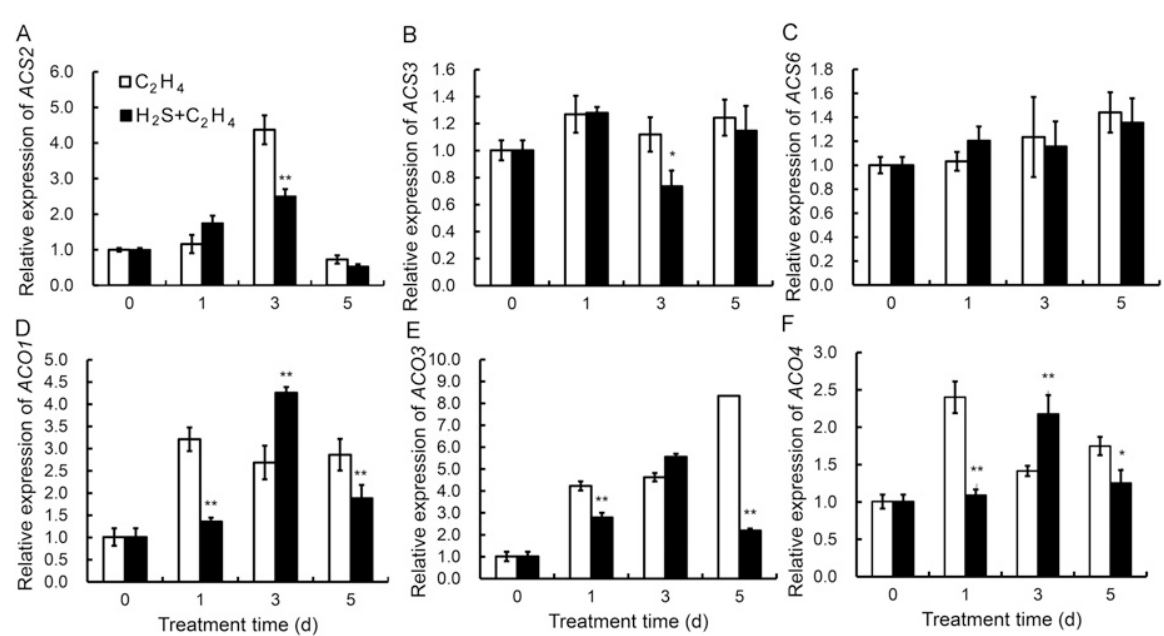

Fig. 4. Changes in the gene expression of ethylene biosynthesis-related genes of 1-aminocyclopropane-1carboxylate synthase [SlACS2 (A), SlACS3 (B), and SlACS6 (C)] and 1-aminocyclopropane-1carboxylate oxidase $[S l A C O 1(\mathbf{D}), \operatorname{SlACO} 3(\mathbf{E})$, and SlACO4 $(\mathbf{F})]$ in tomato fruit during storage after $\mathrm{C}_{2} \mathrm{H}_{4}$ and $\mathrm{C}_{2} \mathrm{H}_{4}+\mathrm{H}_{2} \mathrm{~S}$ treatment for $1 \mathrm{~d}$. Error bars indicate $\mathrm{SE}(\mathrm{n}=3)$. Asterisks indicate significant differences between $\mathrm{C}_{2} \mathrm{H}_{4}$ and $\mathrm{C}_{2} \mathrm{H}_{4}+\mathrm{H}_{2} \mathrm{~S}$ co-treated fruit according to Student's $t$ test $(* P<0.05$; ** $P<0.01)$. 
found to attenuate the expression on day 5 . Figure 5C shows that SIETR3 increased with ethylene treatment on day 1 , followed by a fluctuation. In addition, a significantly lower level of SIETR3 expression with co-treatment compared with ethylene alone was observed on day 1 and day 5 , but the trend was reversed on day 3. Figure 5D shows that SlETR4 expression increased gradually with both treatments; however, a decrease was observed on day 5 with co-treatment. Furthermore, ethylene $+\mathrm{H}_{2} \mathrm{~S}$ treatment induced a higher expression of SIETR4 on day 3 compared with ethylene treatment. Figure $5 \mathrm{E}$ depicts a trend of increasing expression that was observed with both ethylene and ethylene $+\mathrm{H}_{2} \mathrm{~S}$ treatment; however, ethylene $+\mathrm{H}_{2} \mathrm{~S}$ maintained a significantly lower expression level during the entire storage time compared with control. Figure $5 \mathrm{~F}$ shows that ethylene treatment induced an increase of SIETR6 on day 1, followed by a plateau, whereas ethylene $+\mathrm{H}_{2} \mathrm{~S}$ treatment attenuated the expression on day 1 and day 5 . Ethylene $+\mathrm{H}_{2} \mathrm{~S}$ treatment was found to differentially affect the expression of ethylene receptor genes, and the expression levels of SIETRI, SIETR2, SIETR3, SIETR5, and SIETR6 were significantly downregulated at day 5 in the cotreatment group.

Many ethylene response factors become involved in fruit ripening after sensing the signal of ethylene. As shown in Fig. 6A, ethylene treatment induced an increased expression of the ethylene responsive transcription factor $S l C R F 2$ on day 1 , followed by a plateau, whereas ethylene $+\mathrm{H}_{2} \mathrm{~S}$ treatment significantly inhibited the expression on day 1 and day 3. Figure $6 \mathrm{~B}$ shows that SlERF1 increased slightly with both treatments; however, no significant difference was observed. Generally, the trend of increased expression of SIERF2D was observed with both treatments, but $\mathrm{H}_{2} \mathrm{~S}$ caused no significant changes in the expression (Fig. 6C). Figure $6 \mathrm{D}$ shows that $S I E R F 2$ changed little during storage with both treatments, but ethylene $+\mathrm{H}_{2} \mathrm{~S}$ treatment maintained a significantly lower level of expression during the whole storage time.

Correlation analysis and heatmap of the values determined in tomatoes during storage. To determine the factors contributing to tomato fruit softening, the correlation among the value of firmness, activities of cellulase, polygalacturonase, pectin lyase, expressions of softening-related genes (SILAT56, SILAT59, SIPMEU1, and SIPG2), ethylene biosynthesis genes (SlACS2, SlACS3, SlACS6, SlACO1, SlACO3, and SlACO4), ethylene receptor genes (SlETR1, SlETR2, SIETR3, SlETR4, SlETR5, and SIETR6), and ethylene response factors (SlCRF2, SlERF1, SlERFD2, SlERF2) were analyzed. As shown in Fig. 7, the data indicated that fruit firmness was negatively correlated with the expression of ethylenerelated genes SlACOs, SlACSS, SlETRS, and SlERFs in tomato fruits. Similarly, tomato fruit firmness was negatively correlated with the expression of softening-related genes SlLAT56, SILAT59, and SIPG2.
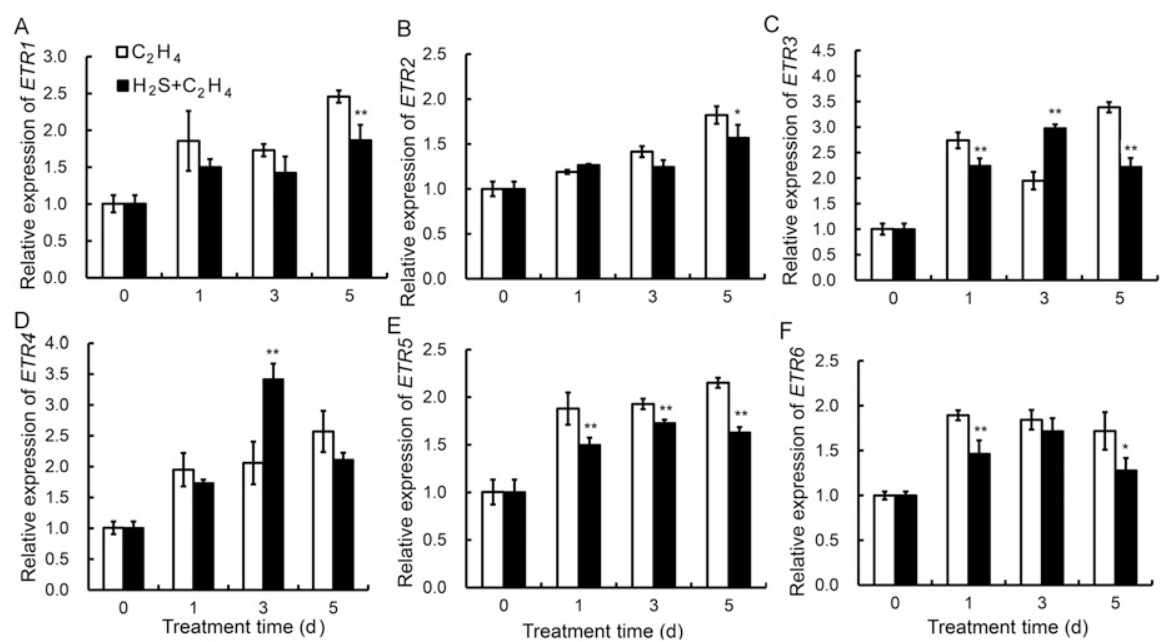

Fig. 5. Changes in the gene expression of ethylene receptor-related genes SIETR1 (A), SIETR2 (B), SIETR3 (C), SIETR4 (D), SIETR5 (E), and SIETR6 (F) in tomato fruit during storage after $\mathrm{C}_{2} \mathrm{H}_{4}$ and $\mathrm{C}_{2} \mathrm{H}_{4}+\mathrm{H}_{2} \mathrm{~S}$ treatment for $1 \mathrm{~d}$. Error bars indicate $\mathrm{SE}(\mathrm{n}=3)$. Asterisks indicate significant differences between fruit treated with $\mathrm{C}_{2} \mathrm{H}_{4}$ and $\mathrm{C}_{2} \mathrm{H}_{4}+\mathrm{H}_{2} \mathrm{~S}$ according to Student's $t$ test $(* P<0.05$; $* * P<0.01)$.
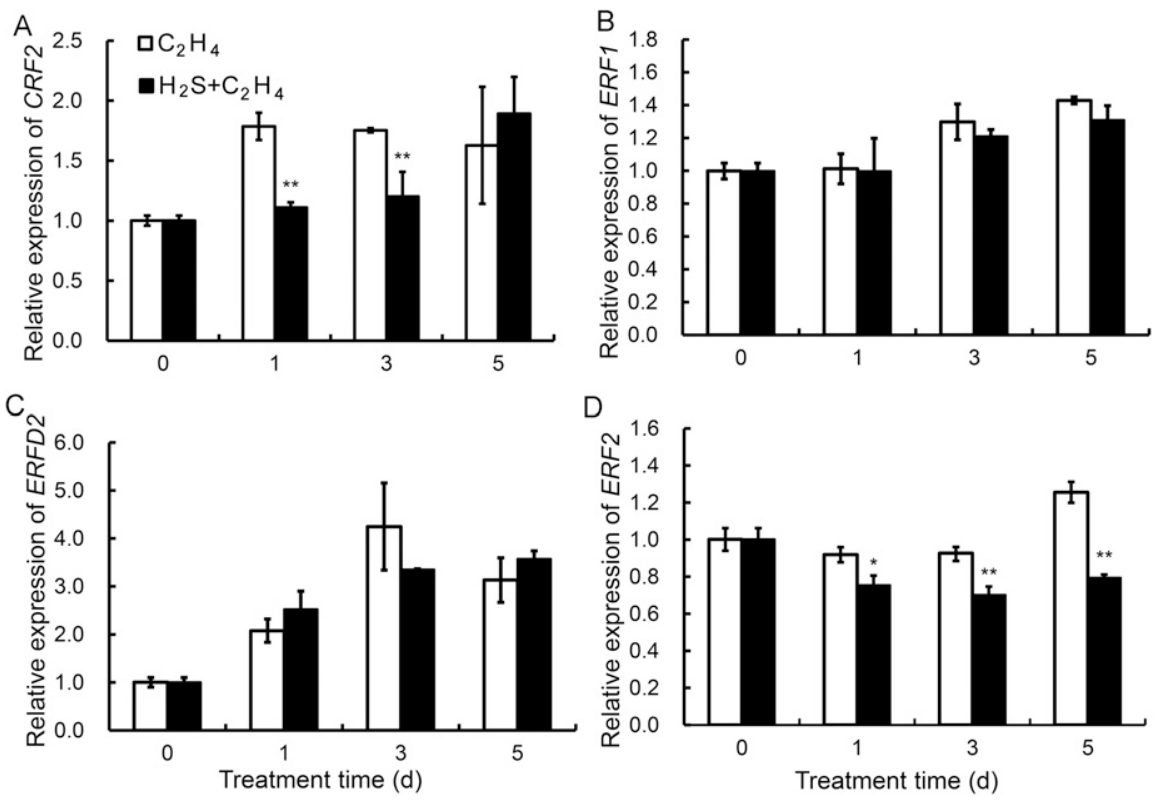

Fig. 6. Changes in the gene expression of ethylene response factors $\operatorname{SlCRF2}$ (A), SlERF1 (B), SlERFD2 (C), and SlERF2 (D) in tomato fruit during storage after $\mathrm{C}_{2} \mathrm{H}_{4}$ and $\mathrm{C}_{2} \mathrm{H}_{4}+\mathrm{H}_{2} \mathrm{~S}$ treatment for $1 \mathrm{~d}$. Error bars indicate SE $(n=3)$. Asterisks indicate significant differences between $\mathrm{C}_{2} \mathrm{H}_{4}$ and $\mathrm{C}_{2} \mathrm{H}_{4}+\mathrm{H}_{2} \mathrm{~S}$ cotreated fruit according to Student's $t$ test $(* P<0.05 ; * * P<0.01)$.

The heatmap of the physiological parameters of tomatoes during storage was drawn (Fig. 8) to show the difference between ethylene and ethylene $+\mathrm{H}_{2} \mathrm{~S}$ treatment. It could be observed that ethylene $+\mathrm{H}_{2} \mathrm{~S}$ alleviated the softening of tomatoes by attenuating the activities of cell wall-degrading enzymes and their expression, and also the expression of ethylene biosynthesis and signaling genes.

\section{Discussion}

Fruit ripening is a complex developmental process that is coordinated with the activities of softening-related enzymes and the upregulation of a large set of ripening-related genes. It has been reported that fruit softening is a significant characteristic of fruit ripeness and an important factor affecting tomato fruit quality, storage, and shelf life (Oms-Oliu et al., 2011). Our results showed that the treatment of tomato fruit with exogenous $\mathrm{H}_{2} \mathrm{~S}$ significantly delayed the fruit softening process and inhibited the activity of softening enzymes. The transition of tomato color from green to red was delayed by $\mathrm{H}_{2} \mathrm{~S}$ compared with ethylene alone. Tomatoes were fumigated with $\mathrm{H}_{2} \mathrm{~S}$ released from $1 \mathrm{~mm}$ of $\mathrm{NaHS}$ or NaHS+ethylene for $1 \mathrm{~d}$ and subjected to normal conditions. According to our previous studies, the NaHS concentration used in the present research was within the safety range. 


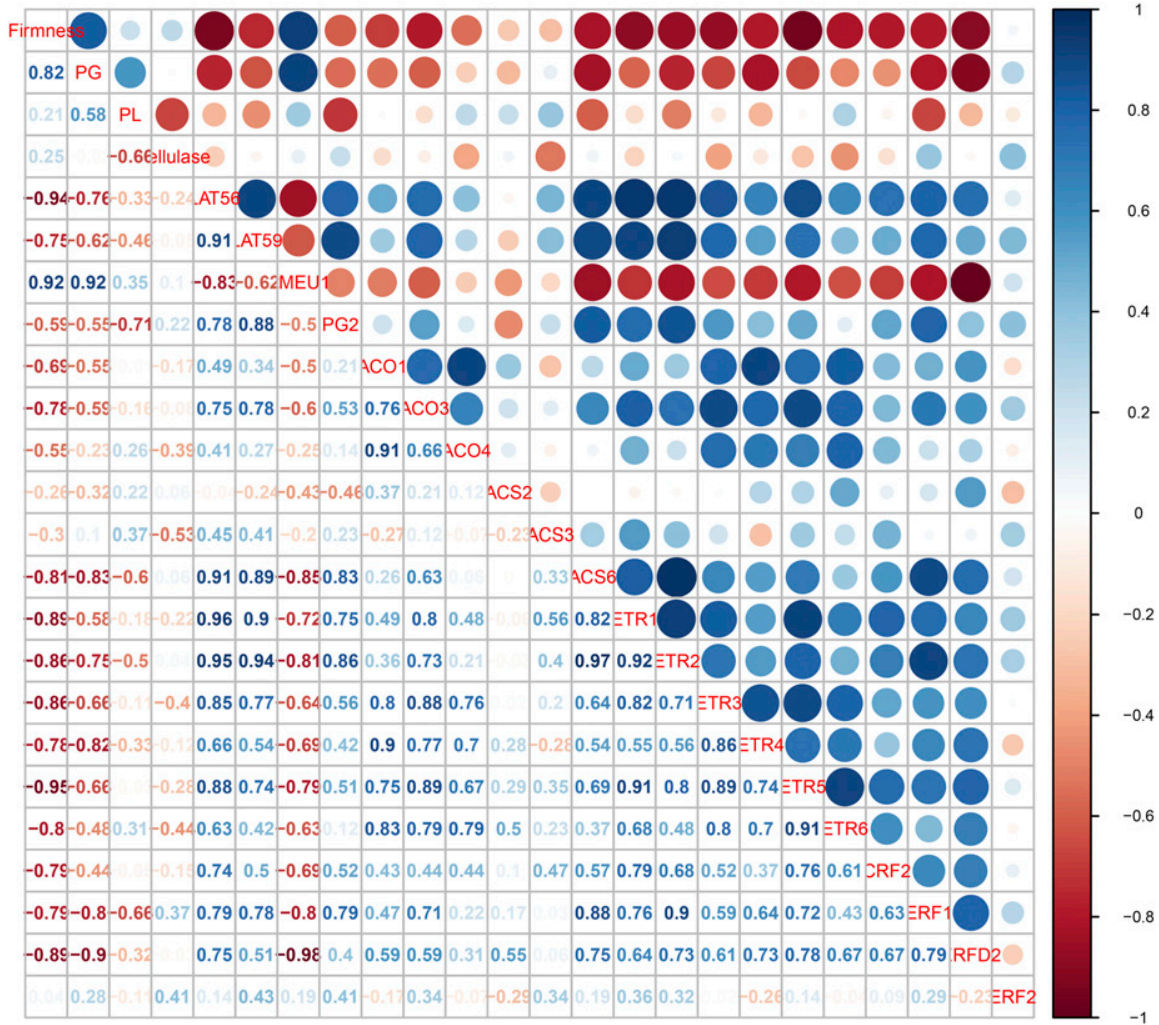

Fig. 7. Correlation analysis among the value of firmness, activities of cellulase, polygalacturonase (PG), pectin lyase (PL), expressions of softening-related genes (SlLAT56, SLLAT59, SIPMEU1, and SlPG2), ethylene biosynthesis genes (SlACS2, SIACS3, SlACS6, SlACO1, SlACO3, and SlACO4), ethylene receptor genes (SIETR1, SIETR2, SIETR3, SIETR4, SIETR5, and SIETR6), and ethylene response factors (SlCRF2, SlERF1, SlERFD2, SlERF2) in tomato fruit treated with $\mathrm{C}_{2} \mathrm{H}_{4}$ and $\mathrm{C}_{2} \mathrm{H}_{4}+\mathrm{H}_{2} \mathrm{~S}$. Pearson's correlation coefficient among data were analyzed using R scripts.

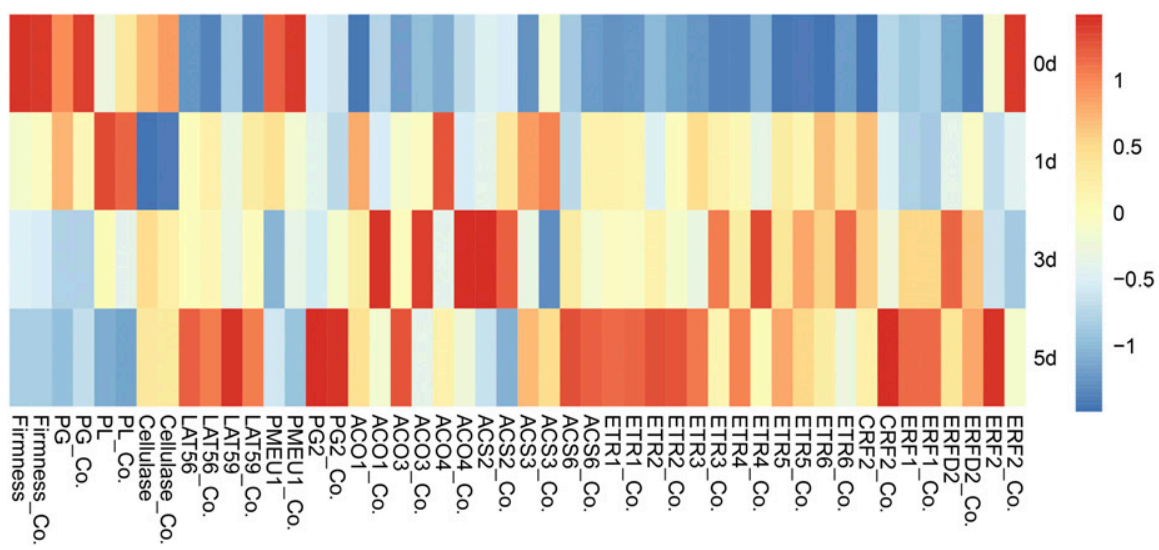

Fig. 8. Heatmap based on the value of firmness, activities of cellulase, polygalacturonase (PG), pectin lyase (PL), expressions of softening-related genes (SILAT56, SILAT59, SIPMEU1, and SIPG2), ethylene biosynthesis genes (SlACS2, SlACS3, SlACS6, SlACO1, SlACO3, and SlACO4), ethylene receptor genes (SIETR1, SIETR2, SIETR3, SIETR4, SIETR5, and SIETR6), and ethylene response factors (SlCRF2, SlERF1, SlERFD2, SlERF2) in tomato fruit treated with $\mathrm{C}_{2} \mathrm{H}_{4}$ and $\mathrm{C}_{2} \mathrm{H}_{4}+\mathrm{H}_{2} \mathrm{~S}$. Co. indicates treatment with $\mathrm{C}_{2} \mathrm{H}_{4}+\mathrm{H}_{2} \mathrm{~S}$. Heatmap scale bars are shown in the right part of the figure. Pearson's correlation coefficient among data were analyzed using R scripts.

Many enzymes are associated with softening in fruits, including pectinmethylesterase, polygalacturonase, cellulase, and others (Brummell and Harpster, 2001). Polygalacturonase and cellulase are required for the modification of pectin and cellulase, respectively. To alleviate fruit softening, various postharvest measures have been applied, such as ultraviolet-C illumination (Bu et al.,
(SILAT56 and SILAT59), pectin methylesterase (SIPMEU1), and polygalacturonase (SlPG2) were downregulated in tomato fruit treated with $\mathrm{H}_{2} \mathrm{~S}$ for $1 \mathrm{~d}$. Among the enzymes, PG showed very high activity during tomato fruit softening, suggesting that PG might be the key enzyme for cell wall degradation (Brummell and Labavitch, 1997). It was found that the gene SlPG2 was significantly upregulated at a late stage of storage, whereas $\mathrm{H}_{2} \mathrm{~S}$ inhibited the increase (Fig. 3D). The results implied that exogenous $\mathrm{H}_{2} \mathrm{~S}$ alleviated tomato fruit ripening by attenuating the activities of cell wall degradation enzymes and their gene expressions.

Tomato is typically a climacteric fruit, and its ripening and senescence are accompanied by the synthesis of large amounts of ethylene. Therefore, the control of ethylene biosynthesis or its signaling pathway can be applied to alleviate tomato fruit ripening and senescence. For instance, 1-MCP, an ethylene receptor inhibitor, is broadly used during postharvest fruit storage (Song et al., 2018; Watkins et al., 2000), and acetylsalicylic acid shows inhibitory effects on ethylene biosynthesis and signaling during kiwifruit ripening (Yin et al., 2013). In the present research, we studied the regulatory role of exogenous $\mathrm{H}_{2} \mathrm{~S}$ on the expression of ethylene synthesis and signal transduction-related genes in tomatoes. ACC synthase and ACC oxidase are the enzymes that catalyze the conversion of AdoMet to ACC and ACC to ethylene, respectively. It was found that $\mathrm{H}_{2} \mathrm{~S}$ significantly reduced the expression of SlACS2 and SlACS3, whereas SlACS4 genes remained unchanged compared with ethylene. Ethylene significantly upregulates the expression of SlACO1, SlACO3, and SlACO4 after $1 \mathrm{~d}$ of treatment, whereas $\mathrm{H}_{2} \mathrm{~S}$ attenuated the increase, suggesting that $\mathrm{H}_{2} \mathrm{~S}$ might inhibit ethylene synthesis at the expression level. These results were consistent with those of our previous report of the role of $\mathrm{H}_{2} \mathrm{~S}$ in alleviating the ripening and senescence of kiwifruit and banana (Ge et al., 2017; Li et al., 2017). The expression patterns of ethylene receptor genes showed that $\mathrm{H}_{2} \mathrm{~S}$ treatment differentially affected their expressions, and expression levels of SIETR1, SIETR2, SIETR3, SIETR5, and SIETR6 were significantly downregulated by $\mathrm{H}_{2} \mathrm{~S}$. After being recognized by ethylene receptors and a signaling cascade, ethylene has physiological roles in a large family of ERFs, subsequently inducing changes in the metabolism involved in fruit ripening and senescence (Bapat et al., 2010). The present results showed that $S I C R F 2$ and SlERF2 were downregulated by $\mathrm{H}_{2} \mathrm{~S}$ treatment. Exogenous $\mathrm{H}_{2} \mathrm{~S}$ was found to antagonize the synthesis and signaling of ethylene, thereby alleviating fruit ripening in tomatoes.

Texture softening is a predominant characteristic of ethylene-induced fruit ripening. We found that $\mathrm{H}_{2} \mathrm{~S}$ could attenuate the expression of ethylene signaling and pathway genes. The cell wall degradation enzymes (CWDE) was also decreased by $\mathrm{H}_{2} \mathrm{~S}$ treatment. Therefore, the correlation was analyzed and the results showed that fruit 
firmness was negative correlated with the expression of ethylene-related genes SlACOs, SlACSs, SlETRs, and SlERFs in tomato fruits. It is difficult to say whether $\mathrm{H}_{2} \mathrm{~S}$ regulates these two events separately or in a linear way. Accumulating reports found that ethylene can act upstream of CWDE. For instance, the expression of CWDE is ethylene-dependent; therefore, fruit softening and cell wall disassembly are regulated by ethylene in Charentais melon (Nishiyama et al., 2007). The ERF DkERFs in persimmon fruit was found to directly bind to the promoter of a CWDE gene DkXTH9 and regulate its gene expression (Wang et al., 2017). Therefore, the role of $\mathrm{H}_{2} \mathrm{~S}$ in regulating fruit softening could be as a linear signaling pathway $\left(\mathrm{H}_{2} \mathrm{~S}\right.$-ethylene-CWDE).

Short-term application of $\mathrm{H}_{2} \mathrm{~S}$ alleviates fruit softening by inhibiting the activities and gene expressions of cell wall-degrading enzymes. This study also demonstrated that $\mathrm{H}_{2} \mathrm{~S}$ downregulates ethylene biosynthesis and signaling genes, effectively protecting cell wall components from being degraded. This work provides insight into the multifaceted role of $\mathrm{H}_{2} \mathrm{~S}$ in fruit senescence and implicates the potential application of $\mathrm{H}_{2} \mathrm{~S}$ during postharvest fruit storage.

\section{Literature Cited}

Abeles, F.B. and C.L. Biles. 1991. Cellulase activity in developing apple fruits. Scientia Hort. 47:77-87.

Adams, D.O. and S.F. Yang. 1979. Ethylene biosynthesis: Identification of 1-aminocyclopropane1-carboxylic acid as an intermediate in the conversion of methionine to ethylene. Proc. Natl. Acad. Sci. USA 76:170-174.

Alexander, L. and D. Grierson. 2002. Ethylene biosynthesis and action in tomato: A model for climacteric fruit ripening. J. Expt. Bot. 53:2039-2055.

Bapat, V.A., P.K. Trivedi, A. Ghosh, V.A. Sane, T.R. Ganapathi, and P. Nath. 2010. Ripening of fleshy fruit: Molecular insight and the role of ethylene. Biotechnol. Adv. 28:94-107.

Beecher, G.R. 1998. Nutrient content of tomatoes and tomato products. Proc. Soc. Exp. Biol. Med. 218:98-100.

Brummell, D.A. and M.H. Harpster. 2001. Cell wall metabolism in fruit softening and quality and its manipulation in transgenic plants. Plant Mol. Biol. 47:311-340.

Brummell, D.A. and J.M. Labavitch. 1997. Effect of antisense suppression of endopolygalacturonase activity on polyuronide molecular weight in ripening tomato fruit and in fruit homogenates. Plant Physiol. 115:717-725.

Bu, J., Y. Yu, G. Aisikaer, and T. Ying. 2013. Postharvest UV-C irradiation inhibits the production of ethylene and the activity of cell walldegrading enzymes during softening of tomato (Lycopersicon esculentum L.) fruit. Postharvest Biol. Technol. 86:337-345.

Chen, J., F.H. Wu, W.H. Wang, C.J. Zheng, G.H. Lin, X.J. Dong, J.X. He, Z.M. Pei, and H.L. Zheng. 2011. Hydrogen sulphide enhances photosynthesis through promoting chloroplast biogenesis, photosynthetic enzyme expression, and thiol redox modification in Spinacia oleracea seedlings. J. Expt. Bot. 62:4481-4493.

De Boer, K., S. Tilleman, L. Pauwels, R. Vanden Bossche, V. De Sutter, R. Vanderhaeghen, P. Hilson, J.D. Hamill, and A. Goossens. 2011. APETALA2/ETHYLENE RESPONSE FACTOR and basic helix-loop-helix tobacco transcrip- tion factors cooperatively mediate jasmonateelicited nicotine biosynthesis. Plant J. 66:1053-1065.

Fischer, R.L. and A.B. Bennett. 1991. Role of cell wall hydrolases in fruit ripening. Annu. Rev. Plant Physiol. Plant Mol. Biol. 42:675-703.

Gadalla, M.M. and S.H. Snyder. 2010. Hydrogen sulfide as a gasotransmitter. J. Neurochem. 113:14-26.

Gao, S.P., K.D. Hu, L.Y. Hu, Y.H. Li, Y. Han, H.L. Wang, K. Lv, Y.S. Liu, and H. Zhang. 2013. Hydrogen sulfide delays postharvest senescence and plays an antioxidative role in freshcut kiwifruit. HortScience 48:1385-1392.

Ge, Y., K.D. Hu, S.S. Wang, L.Y. Hu, X.Y. Chen, Y.H. Li, Y. Yang, F. Yang, and H. Zhang. 2017. Hydrogen sulfide alleviates postharvest ripening and senescence of banana by antagonizing the effect of ethylene. PLoS One 12:E0180113.

Giovannoni, J.J. 2007. Fruit ripening mutants yield insights into ripening control. Curr. Opin. Plant Biol. 10:283-289.

Grierson, D., M.J. Maunders, A. Slater, J. Ray, C.R. Bird, W. Schuch, M.J. Holdsworth, G.A. Tucker, and J.E. Knapp. 1986. Gene expression during tomato ripening. Philos. Trans. R. Soc. Lond. B Biol. Sci. 314:399-410.

Gwanpua, S.G., I. Mellidou, J. Boeckx, C. Kyomugasho, N. Bessemans, B.E. Verlinden, M.L.A.T.M. Hertog, M. Hendrickx, B.M. Nicolai, and A.H. Geeraerd. 2016. Expression analysis of candidate cell wall-related genes associated with changes in pectin biochemistry during postharvest apple softening. Postharvest Biol. Technol. 112:176-185.

Hu, L.Y., S.L. Hu, J. Wu, Y.H. Li, J.L. Zheng, Z.J. Wei, J. Liu, H.L. Wang, Y.S. Liu, and H. Zhang. 2012. Hydrogen sulfide prolongs postharvest shelf life of strawberry and plays an antioxidative role in fruits. J. Agr. Food Chem. 60:8684-8693.

Li, T.T., Z.R. Li, K.D. Hu, L.Y. Hu, X.Y. Chen, Y.H. Li, Y. Yang, F. Yang, and H. Zhang. 2017. Hydrogen sulfide alleviates kiwifruit ripening and senescence by antagonizing effect of ethylene. HortScience 52:1556-1562.

Licausi, F., M. Ohme-Takagi, and P. Perata. 2013. APETALA2/Ethylene Responsive Factor (AP2/ERF) transcription factors: Mediators of stress responses and developmental programs. New Phytol. 199:639-649.

Lisjak, M., N. Srivastava, T. Teklic, L. Civale, K. Lewandowski, I. Wilson, M.E. Wood, M. Whiteman, and J.T. Hancock. 2010. A novel hydrogen sulfide donor causes stomatal opening and reduces nitric oxide accumulation. Plant Physiol. Biochem. 48:931-935.

Liu, M., J. Pirrello, C. Chervin, J.P. Roustan, and M. Bouzayen. 2015. Ethylene control of fruit ripening: Revisiting the complex network of transcriptional regulation. Plant Physiol. 169:2380-2390.

Musse, M., S. Quellec, M. Cambert, M.F. Devaux, M. Lahaye, and F. Mariette. 2009. Monitoring the postharvest ripening of tomato fruit using quantitative MRI and NMR relaxometry. Postharvest Biol. Technol. 53:22-35.

Nishiyama, K., M. Guis, J.K. Rose, Y. Kubo, K.A. Bennett, L. Wangjin, K. Kato, K. Ushijima, R. Nakano, A. Inaba, M. Bouzayen, A. Latche, J.C. Pech, and A.B. Bennett. 2007. Ethylene regulation of fruit softening and cell wall disassembly in Charentais melon. J. Expt. Bot. 58:1281-1290.

Oms-Oliu, G., M.L.A.T.M. Hertog, B. Van de. Poel, J. Ampofo-Asiama, A.H. Geeraerd, and B.M. Nicolaii. 2011. Metabolic characterization of tomato fruit during preharvest development, ripening, and postharvest shelf-life. Postharvest Biol. Technol. 62:7-16.
Pathak, N. and G.G. Sanwal. 1998. Multiple forms of polygalacturonase from banana fruits. Phytochemistry 48:249-255.

Payton, S., R.G. Fray, S. Brown, and D. Grierson. 1996. Ethylene receptor expression is regulated during fruit ripening, flower senescence and abscission. Plant Mol. Biol. 31:1227-1231.

Pitt, D. 1988. Pectin lyase from Phoma medicaginis var. pinodella. Methods Enzymol. 161:350-354.

Qi, W., F. Sun, Q. Wang, M. Chen, Y. Huang, Y.Q. Feng, X. Luo, and J. Yang. 2011. Rice ethyleneresponse AP2/ERF factor OsEATB restricts internode elongation by down-regulating a gibberellin biosynthetic gene. Plant Physiol. 157:216-228.

Qiao, Z., J. Tao, Z. Liu, L. Zhang, Z. Jin, D. Liu, and Y. Pei. 2015. $\mathrm{H}_{2} \mathrm{~S}$ acting as a downstream signaling molecule of SA regulates Cd tolerance in Arabidopsis. Plant Soil 393:137-146.

Sisler, E.C. and S.F. Yang. 1984. Ethylene, the gaseous plant hormone. Bioscience 34:234-238.

Song, J.S., S.K. Yoo, and D.S. Kim. 2018. The effect of 1-(3-phenyl-propyl)cyclopropene versus $1-\mathrm{MCP}$ on the quality and storage life of tomato (Solanum lycopersicum) fruit. Postharvest Biol. Technol. 145:20-26.

Thirugnanasambantham, K., S. Durairaj, S. Saravanan, K. Karikalan, S. Muralidaran, and V.I.H. Islam. 2015. Role of ethylene response transcription factor (ERF) and its regulation in response to stress encountered by plants. Plant Mol. Biol. Rpt. 33:347-357.

Thompson, D.S., W.J. Davies, and L.C. Ho. 1998. Regulation of tomato fruit growth by epidermal cell wall enzymes. Plant Cell Environ. 21:589-599.

Wang, L.C.K., H. Li, and J.R. Ecker. 2002. Ethylene biosynthesis and signaling networks. Plant Cell 14(Suppl):S131-S151.

Wang, M.M., Q.G. Zhu, C.L. Deng, Z.R. Luo, N.J. Sun, D. Grierson, X.R. Yin, and K.S. Chen. 2017. Hypoxia-responsive ERFs involved in postdeastringency softening of persimmon fruit. Plant Biotechnol. J. 15:1409-1419.

Wang, R. 2010. Hydrogen sulfide: The third gasotransmitter in biology and medicine. Antioxid. Redox Signal. 12:1061-1064.

Wang, Y., L. Le, W. Cui, X. Sheng, W. Shen, and W. Ren. 2012. Hydrogen sulfide enhances alfalfa (Medicago sativa) tolerance against salinity during seed germination by nitric oxide pathway. Plant Soil 351:107-119.

Watkins, C.B., J.F. Nock, and B.D. Whitaker. 2000. Responses of early, mid and late season apple cultivars to postharvest application of 1methylcyclopropene (1-MCP) under air and controlled atmosphere storage conditions. Postharvest Biol. Technol. 19:17-32.

Yin, X.R., Y. Zhang, B. Zhang, S.L. Yang, Y.N Shi, I.B. Ferguson, and K.S. Chen. 2013. Effects of acetylsalicylic acid on kiwifruit ethylene biosynthesis and signaling components. Postharvest Biol. Technol. 83:27-33.

Zaharah, S.S. and Z. Singh. 2011. Mode of action of nitric oxide in inhibiting ethylene biosynthesis and fruit softening during ripening and cool storage of 'Kensington Pride' mango. Postharvest Biol. Technol. 62:258-266.

Zhai, R., J. Liu, F. Liu, Y. Zhao, L. Liu, F. Chen, H. Wang, X. Li, Z. Wang, and F. Ma. 2018. Melatonin limited ethylene production, softening and reduced physiology disorder in pear (Pyrus communis L.) fruit during senescence. Postharvest Biol. Technol. 139:38-46.

Zhang, H., J. Tang, X.P. Liu, Y. Wang, W. Yu, W.Y. Peng, F. Fang, D.F. Ma, Z.J. Wei, and L.Y. Hu. 2009. Hydrogen sulfide promotes root organogenesis in Ipomoea batatas, Salix matsudana and Glycine max. J. Integr. Plant Biol. 51:1086-1094. 